\title{
Inclusion and Neighborhood Properties for Certain Classes of Multivalently Analytic Functions
}

\begin{abstract}
Serap Bulut
Civil Aviation College, Kocaeli University, Arslanbey Campus, İzmit, 41285 Kocaeli, Turkey

Correspondence should be addressed to Serap Bulut; bulutserap@yahoo.com

Received 10 May 2013; Accepted 5 October 2013

Academic Editor: Lianzhong Yang

Copyright ( 2013 Serap Bulut. This is an open access article distributed under the Creative Commons Attribution License, which permits unrestricted use, distribution, and reproduction in any medium, provided the original work is properly cited.

We introduce and investigate two new general subclasses of multivalently analytic functions of complex order by making use of the familiar convolution structure of analytic functions. Among the various results obtained here for each of these function classes, we derive the coefficient inequalities and other interesting properties and characteristics for functions belonging to the classes introduced here.
\end{abstract}

\section{Introduction and Definitions}

Let $\mathbb{R}=(-\infty, \infty)$ be the set of real numbers, let $\mathbb{C}$ be the set of complex numbers, let $\mathbb{N}=\{1,2, \ldots\}$ be the set of positive integers, and let $\mathbb{N}_{0}=\mathbb{N} \cup\{0\}$.

Let $\mathscr{T}_{p}$ denote the class of functions of the form

$$
\begin{array}{r}
f(z)=z^{p}-\sum_{j=k}^{\infty} a_{j} z^{j} \\
\left(p<k ; a_{j} \geq 0(j \geq k) ; k, p \in \mathbb{N}\right),
\end{array}
$$

which are analytic and $p$-valent in the open unit disk

$$
\mathbb{U}=\{z \in \mathbb{C}:|z|<1\} .
$$

Denote by $f * g$ the Hadamard product (or convolution) of the functions $f$ and $g$; that is, if $f$ is given by (1) and $g$ is given by

$$
\begin{array}{r}
g(z)=z^{p}+\sum_{j=k}^{\infty} b_{j} z^{j} \\
\left(p<k ; b_{j} \geq 0(j \geq k) ; k, p \in \mathbb{N}\right),
\end{array}
$$

then

$$
(f * g)(z):=z^{p}-\sum_{j=k}^{\infty} a_{j} b_{j} z^{j}=:(g * f)(z) .
$$

Definition 1. Let the function $f \in \mathscr{T}_{p}$. Then one says that $f$ is in the class $\mathcal{S}_{g}(p, k, \lambda, \mu, b, \beta, m, n)$ if it satisfies the condition

$$
\begin{aligned}
& \left|\frac{1}{b}\left(\frac{z^{n}\left(\mathscr{F}_{\lambda, \mu} * g\right)^{(m+n)}(z)}{\left(\mathscr{F}_{\lambda, \mu} * g\right)^{(m)}(z)}-(p-m)_{n}\right)\right|<\beta \\
& \left(m+n<p<k ; p, n \in \mathbb{N} ; m \in \mathbb{N}_{0} ; b \in \mathbb{C} \backslash\{0\} ;\right. \\
& 0 \leq \mu \leq \lambda \leq 1 ; 0<\beta \leq 1 ; z \in \mathbb{U}),
\end{aligned}
$$

where

$$
\begin{aligned}
& \mathscr{F}_{\lambda, \mu}(z) \\
& =\lambda \mu z^{2} f^{\prime \prime}(z)+(\lambda-\mu) z f^{\prime}(z)+(1-\lambda+\mu) f(z),
\end{aligned}
$$

$g$ is given by (3), and $(\nu)_{n}$ denotes the falling factorial defined as follows:

$$
(\nu)_{0}=1=:\left(\begin{array}{l}
\nu \\
0
\end{array}\right),
$$

$$
(\nu)_{n}=v(\nu-1) \cdots(\nu-n+1)=: n !\left(\begin{array}{l}
\nu \\
n
\end{array}\right) \quad(n \in \mathbb{N}) .
$$

Various special cases of the class $\delta_{g}(p, k, \lambda, \mu, b, \beta, m, n)$ were considered by many earlier researchers on this topic of Geometric Function Theory. For example, $\delta_{g}(p, k, \lambda, \mu, b$, $\beta, m, n)$ reduces to the function class 
(i) for $m=0, n=1$, and $\mu=0$, studied by Mostafa and Aouf [1];

(ii) for $\lambda=\mu=0$ and $\beta=1$, studied by Srivastava et al. $[2]$;

(iii) for $n=1, \lambda=\mu=0$, and $\beta=1$, studied by Prajapat et al. [3];

(iv) for $m=0, n=1, \beta=1$, and $b=p(1-\alpha)(0 \leq \alpha<$ $1)$, studied by Srivastava and Bulut [4];

(v) for $m=0, n=1, \lambda=\mu=0, \beta=1$ and $b=p(1-$ $\alpha)(0 \leq \alpha<1)$, studied by Ali et al. [5].

Definition 2. Let the function $f \in \mathscr{T}_{p}$. Then one says that $f$ is in the class $\mathscr{P}_{g}(p, k, \lambda, \mu, b, \beta, m, n ; \gamma)$ if it satisfies the condition

$$
\begin{aligned}
& \mid \frac{1}{b}\left((p)_{n}(1-\gamma)\left(\frac{\left(\mathscr{F}_{\lambda, \mu} * g\right)(z)}{z^{n}}\right)\right. \\
& \left.\quad+\gamma\left(\mathscr{F}_{\lambda, \mu} * g\right)^{(m+n)}(z)-(p-m)_{n}\right) \mid \\
& \quad<(p-m)_{n} \\
& \left(m+n<p<k ; p, n \in \mathbb{N} ; m \in \mathbb{N}_{0} ; b \in \mathbb{C} \backslash\{0\} ;\right. \\
& \quad 0 \leq \mu \leq \lambda \leq 1 ; \gamma \geq 0 ; z \in \mathbb{U}),
\end{aligned}
$$

where $g$ and $\mathscr{F}_{\lambda, \mu}$ are defined by (3) and (6), respectively.

Setting $\lambda=\mu=0, \beta=1$ in Definition 2, we have the special class (which generalizes the class defined by Prajapat et al. [3]) introduced by Srivastava et al. [2].

Following a recent investigation by Frasin and Darus [6], if $f \in \mathscr{T}_{p}$ and $\delta \geq 0$, then we define the $(q, \delta)$-neighborhood of the function $f$ by

$$
\begin{aligned}
& \mathcal{N}_{k, \delta}^{q}(f)=\left\{h \in \mathscr{T}_{p}: h(z)=z^{p}-\sum_{j=k}^{\infty} c_{j} z^{j},\right. \\
& \left.\sum_{j=k}^{\infty} j^{q+1}\left|a_{j}-c_{j}\right| \leq \delta\right\} .
\end{aligned}
$$

It follows from the definition (9) that if

$$
e(z)=z^{p} \quad(p \in \mathbb{N})
$$

then

$$
\begin{aligned}
\mathscr{N}_{k, \delta}^{q}(e)= & \left\{h \in \mathscr{T}_{p}: h(z)=z^{p}-\sum_{j=k}^{\infty} c_{j} z^{j},\right. \\
& \left.\sum_{j=k}^{\infty} j^{q+1}\left|c_{j}\right| \leq \delta\right\} .
\end{aligned}
$$

The main object of this paper is to investigate the various properties and characteristics of functions belonging to the above-defined classes

$$
\mathcal{S}_{g}(p, k, \lambda, \mu, b, \beta, m, n), \quad \mathscr{P}_{g}(p, k, \lambda, \mu, b, \beta, m, n ; \gamma) .
$$

Apart from deriving coefficient bounds and coefficient inequalities for each of these classes, we establish several inclusion relationships involving the $(q, \delta)$-neighborhoods of functions belonging to the general classes which are introduced above.

\section{Coefficient Bounds and \\ Coefficient Inequalities}

We begin by proving a necessary and sufficient condition for the function $f \in \mathscr{T}_{p}$ to be in each of the classes

$$
\mathcal{S}_{g}(p, k, \lambda, \mu, b, \beta, m, n), \quad \mathscr{P}_{g}(p, k, \lambda, \mu, b, \beta, m, n ; \gamma) .
$$

Theorem 3. Let the function $f \in \mathscr{T}_{p}$ be given by (1). Then $f$ is in the class $\mathcal{S}_{g}(p, k, \lambda, \mu, b, \beta, m, n)$ if and only if

$$
\begin{aligned}
\sum_{j=k}^{\infty}(j)_{m}\left[(j-m)_{n}-(p-m)_{n}+\beta|b|\right] \psi(j) a_{j} b_{j} \\
\leq \beta|b|(p)_{m} \psi(p) \\
\left(m+n<p<k ; p, n \in \mathbb{N} ; m \in \mathbb{N}_{0} ;\right. \\
b \in \mathbb{C} \backslash\{0\} ; 0<\beta \leq 1 ; z \in \mathbb{U}),
\end{aligned}
$$

where

$$
\psi(s)=(s-1)(\lambda \mu s+\lambda-\mu)+1 \quad(0 \leq \mu \leq \lambda \leq 1) .
$$

Proof. We first suppose that the function $f$ given by (1) is in the class $\mathcal{S}_{g}(p, k, \lambda, \mu, b, \beta, m, n)$. Then, in view of (3)-(6), we have

$$
\operatorname{Re}\left\{\frac{z^{n}\left(\mathscr{F}_{\lambda, \mu} * g\right)^{(m+n)}(z)}{\left(\mathscr{F}_{\lambda, \mu} * g\right)^{(m)}(z)}-(p-m)_{n}\right\}>-\beta|b|
$$

or equivalently

$$
\begin{aligned}
& \operatorname{Re}\left\{\frac{-\sum_{j=k}^{\infty}(j)_{m}\left[(j-m)_{n}-(p-m)_{n}\right] \psi(j) a_{j} b_{j} z^{j-p}}{(p)_{m} \psi(p)-\sum_{j=k}^{\infty}(j)_{m} \psi(j) a_{j} b_{j} z^{j-p}}\right\} \\
& >-\beta|b| .
\end{aligned}
$$

If we choose $z$ to be real and let $z \rightarrow 1^{-}$, we arrive easily at the inequality (14).

Conversely, we suppose that the inequality (14) holds true and let

$$
z \in \partial \mathbb{U}=\{z \in \mathbb{C}:|z|=1\} .
$$


Then, we find that

$$
\begin{aligned}
& \left|\frac{z^{n}\left(\mathscr{F}_{\lambda, \mu} * g\right)^{(m+n)}(z)}{\left(\mathscr{F}_{\lambda, \mu} * g\right)^{(m)}(z)}-(p-m)_{n}\right| \\
& \quad=\left|\frac{\sum_{j=k}^{\infty}(j)_{m}\left[(j-m)_{n}-(p-m)_{n}\right] \psi(j) a_{j} b_{j} z^{j-p}}{(p)_{m} \psi(p)-\sum_{j=k}^{\infty}(j)_{m} \psi(j) a_{j} b_{j} z^{j-p}}\right| \\
& \quad \leq \frac{\sum_{j=k}^{\infty}(j)_{m}\left[(j-m)_{n}-(p-m)_{n}\right] \psi(j) a_{j} b_{j}}{(p)_{m} \psi(p)-\sum_{j=k}^{\infty}(j)_{m} \psi(j) a_{j} b_{j}} \\
& \quad<\frac{\beta|b|\left[(p)_{m} \psi(p)-\sum_{j=k}^{\infty}(j)_{m} \psi(j) a_{j} b_{j}\right]}{(p)_{m} \psi(p)-\sum_{j=k}^{\infty}(j)_{m} \psi(j) a_{j} b_{j}}=\beta|b| .
\end{aligned}
$$

Hence, by the Maximum Modulus Theorem, we have

$$
f \in \mathcal{S}_{g}(p, k, \lambda, \mu, b, \beta, m, n)
$$

which evidently completes the proof of Theorem 3 .

Remark 4. If we set $\lambda=\mu=0$ and $\beta=1$ in Theorem 3, then we have [2, Theorem 1].

Lemma 5. Let the function $f \in \mathscr{T}_{p}$ given by (1) be in the class $\mathcal{S}_{g}(p, k, \lambda, \mu, b, \beta, m, n)$. Then, for $b_{j} \geq b_{k}(j \geq k)$, one has

$$
\begin{array}{r}
\sum_{j=k}^{\infty} a_{j} \leq \frac{\beta|b|(p)_{m} \psi(p)}{(k)_{m}\left[(k-m)_{n}-(p-m)_{n}+\beta|b|\right] \psi(k) b_{k}}, \\
\sum_{j=k}^{\infty} j a_{j} \leq \frac{\beta|b|(p)_{m} \psi(p)(k-m) !}{(k-1) !\left[(k-m)_{n}-(p-m)_{n}+\beta|b|\right] \psi(k) b_{k}} \\
(p>|b|),
\end{array}
$$

where $\psi$ is defined by (15).

Proof. Let $f \in \mathcal{S}_{g}(p, k, \lambda, \mu, b, \beta, m, n)$. Then, in view of the assertion (14), we have

$$
\sum_{j=k}^{\infty} a_{j} \leq \frac{\beta|b|(p)_{m} \psi(p)}{(k)_{m}\left[(k-m)_{n}-(p-m)_{n}+\beta|b|\right] \psi(k) b_{k}} .
$$

Furthermore, by rewriting the assertion (14) as follows:

$$
\begin{aligned}
& \sum_{j=k}^{\infty} \frac{j !}{(j-m) !}\left[(j-m)_{n}-(p-m)_{n}+\beta|b|\right] \psi(j) a_{j} b_{j} \\
& \leq \beta|b|(p)_{m} \psi(p),
\end{aligned}
$$

we obtain

$$
\sum_{j=k}^{\infty} j a_{j} \leq \frac{\beta|b|(p)_{m} \psi(p)(k-m) !}{(k-1) !\left[(k-m)_{n}-(p-m)_{n}+\beta|b|\right] \psi(k) b_{k}} .
$$

Similar to Theorem 3, we can prove the following result.

Theorem 6. Let the function $f \in \mathscr{T}_{p}$ be given by (1). Then $f$ is in the class $\mathscr{P}_{g}(p, k, \lambda, \mu, b, \beta, m, n ; \gamma)$ if and only if

$$
\begin{array}{r}
\sum_{j=k}^{\infty}(j-n)_{m}\left[\gamma(j)_{n}-(\gamma-1)(p)_{n}\right] \psi(j) a_{j} b_{j} \\
\leq(p-m)_{n}\left[|b|-1+(p)_{m} \psi(p)\right] \\
\left(m+n<p<k ; p, n \in \mathbb{N} ; m \in \mathbb{N}_{0} ; b \in \mathbb{C} \backslash\{0\} ;\right. \\
0 \leq \mu \leq \lambda \leq 1 ; \gamma \geq 0 ; z \in \mathbb{U}),
\end{array}
$$

where $\psi$ is defined by (15).

Remark 7. If we set $\lambda=\mu=0$ and $\beta=1$ in Theorem 6 , then we have [2, Theorem 2].

Lemma 8. Let the function $f \in \mathscr{T}_{p}$ given by (1) be in the class $\mathscr{P}_{g}(p, k, \lambda, \mu, b, \beta, m, n ; \gamma)$. Then, for $b_{j} \geq b_{k}(j \geq k)$, one has

$$
\begin{aligned}
& \sum_{j=k}^{\infty} a_{j} \leq \frac{(p-m)_{n}\left[|b|-1+(p)_{m} \psi(p)\right]}{(k-n)_{m}\left[\gamma(k)_{n}-(\gamma-1)(p)_{n}\right] \psi(k) b_{k}}, \\
& \sum_{j=k}^{\infty} j a_{j} \leq \frac{k(p-m)_{n}\left[|b|-1+(p)_{m} \psi(p)\right](k-n-m) !}{(k-n) !\left[\gamma(k)_{n}-(\gamma-1)(p)_{n}\right] \psi(k) b_{k}} \\
& (\gamma>1),
\end{aligned}
$$

where $\psi$ is defined by (15).

Proof. Let $f \in \mathscr{P}_{g}(p, k, \lambda, \mu, b, \beta, m, n ; \gamma)$. Then, in view of the assertion (26), we have

$$
\sum_{j=k}^{\infty} a_{j} \leq \frac{(p-m)_{n}\left[|b|-1+(p)_{m} \psi(p)\right]}{(k-n)_{m}\left[\gamma(k)_{n}-(\gamma-1)(p)_{n}\right] \psi(k) b_{k}} .
$$

Furthermore, we also have from the assertion (26)

$$
\sum_{j=k}^{\infty} j a_{j} \leq \frac{k(p-m)_{n}\left[|b|-1+(p)_{m} \psi(p)\right](k-n-m) !}{(k-n) !\left[\gamma(k)_{n}-(\gamma-1)(p)_{n}\right] \psi(k) b_{k}} .
$$

\section{A Set of Inclusion Relationships}

In this section, we determine inclusion relations for the classes

$$
\mathcal{S}_{g}(p, k, \lambda, \mu, b, \beta, m, n), \quad \mathscr{P}_{g}(p, k, \lambda, \mu, b, \beta, m, n ; \gamma)
$$

involving $(q, \delta)$-neighborhoods defined by (9) and (11). 
Theorem 9. If $b_{j} \geq b_{k}(j \geq k)$ and

$$
\begin{array}{r}
\delta=\frac{\beta|b|(p)_{m} \psi(p)(k-m) !}{(k-1) !\left[(k-m)_{n}-(p-m)_{n}+\beta|b|\right] \psi(k) b_{k}} \\
(p>|b|),
\end{array}
$$

then

$$
\mathcal{S}_{g}(p, k, \lambda, \mu, b, \beta, m, n) \subset \mathcal{N}_{k, \delta}^{0}(e),
$$

where e and $\psi$ are given by (10) and (15), respectively.

Proof. The inclusion relation (33) would follow readily from the definition (11) and the assertion (22).

Remark 10. If we set $\lambda=\mu=0$ and $\beta=1$ in Theorem 9, then we have [2, Theorem 3].

Theorem 11. If $b_{j} \geq b_{k}(j \geq k)$ and

$$
\begin{array}{r}
\delta=\frac{k(p-m)_{n}\left[|b|-1+(p)_{m} \psi(p)\right](k-n-m) !}{(k-n) !\left[\gamma(k)_{n}-(\gamma-1)(p)_{n}\right] \psi(k) b_{k}} \\
(\gamma>1),
\end{array}
$$

then

$$
\mathscr{P}_{g}(p, k, \lambda, \mu, b, \beta, m, n ; \gamma) \subset \mathscr{N}_{k, \delta}^{0}(e)
$$

where e and $\psi$ are given by (10) and (15), respectively.

Proof. The inclusion relation (35) would follow readily from the definition (11) and the assertion (28).

Remark 12. If we set $\lambda=\mu=0$ and $\beta=1$ in Theorem 11, then we have [2, Theorem 4].

\section{Neighborhood Properties}

In this section, we determine the neighborhood properties for each of the function classes

$$
\mathcal{S}_{g}^{(\alpha)}(p, k, \lambda, \mu, b, \beta, m, n), \quad \mathscr{P}_{g}^{(\alpha)}(p, k, \lambda, \mu, b, \beta, m, n ; \gamma)
$$

which are defined as follows.

Definition 13. A function $f \in \mathscr{T}_{p}$ is said to be in the class $\mathcal{S}_{g}^{(\alpha)}(p, k, \lambda, \mu, b, \beta, m, n)$ if there exists a function $h \in$ $\mathcal{S}_{g}(p, k, \lambda, \mu, b, \beta, m, n)$ such that

$$
\left|\frac{f(z)}{h(z)}-1\right|<p-\alpha \quad(0 \leq \alpha<p ; z \in \mathbb{U}) .
$$

Definition 14. A function $f \in \mathscr{T}_{p}$ is said to be in the class $\mathscr{P}_{g}^{(\alpha)}(p, k, \lambda, \mu, b, \beta, m, n ; \gamma)$ if there exists a function $h \in$ $\mathscr{P}_{g}(p, k, \lambda, \mu, b, \beta, m, n ; \gamma)$ such that the inequality (37) holds true.
Setting $\lambda=\mu=0, \beta=1$ in Definitions 13 and 14, we have the special classes

$$
\mathcal{S}_{g}^{(\alpha)}(p, k, b, m, n), \quad \mathscr{P}_{g}^{(\alpha)}(p, k, b, m, n ; \gamma)
$$

introduced by Srivastava et al. [2], respectively.

Theorem 15. If $h \in \mathcal{S}_{g}(p, k, \lambda, \mu, b, \beta, m, n)$ and

$$
\begin{aligned}
\alpha= & p-\frac{\delta}{k^{q+1}} \\
& \times\left((k)_{m}\left[(k-m)_{n}-(p-m)_{n}+\beta|b|\right] \psi(k) b_{k}\right) \\
& \times\left((k)_{m}\left[(k-m)_{n}-(p-m)_{n}+\beta|b|\right]\right. \\
& \left.\times \psi(k) b_{k}-\beta|b|(p)_{m} \psi(p)\right)^{-1},
\end{aligned}
$$

then

$$
\mathcal{N}_{k, \delta}^{q}(h) \subset \mathcal{S}_{g}^{(\alpha)}(p, k, \lambda, \mu, b, \beta, m, n)
$$

where $\psi$ is defined by (15).

Proof. Suppose that $f \in \mathcal{N}_{k, \delta}^{q}(h)$. Then we find from (9) that

$$
\sum_{j=k}^{\infty} j^{q+1}\left|a_{j}-c_{j}\right| \leq \delta
$$

which readily implies that

$$
\sum_{j=k}^{\infty}\left|a_{j}-c_{j}\right| \leq \frac{\delta}{k^{q+1}}
$$

Since $h \in \mathcal{S}_{g}(p, k, \lambda, \mu, b, \beta, m, n)$, we find from (21) that

$$
\sum_{j=k}^{\infty} c_{j} \leq \frac{\beta|b|(p)_{m} \psi(p)}{(k)_{m}\left[(k-m)_{n}-(p-m)_{n}+\beta|b|\right] \psi(k) b_{k}}
$$

so that

$$
\begin{aligned}
& \left|\frac{f(z)}{h(z)}-1\right| \\
& \quad \leq \frac{\sum_{j=k}^{\infty}\left|a_{j}-c_{j}\right|}{1-\sum_{j=k}^{\infty} c_{j}} \\
& \leq \frac{\delta}{k^{q+1}}\left((k)_{m}\left[(k-m)_{n}-(p-m)_{n}+\beta|b|\right] \psi(k) b_{k}\right) \\
& \quad \times\left((k)_{m}\left[(k-m)_{n}-(p-m)_{n}+\beta|b|\right]\right. \\
& \left.\quad \times \psi(k) b_{k}-\beta|b|(p)_{m} \psi(p)\right)^{-1} \\
& =p-\alpha,
\end{aligned}
$$

where $\alpha$ is given by (39). Thus, by Definition 13, $f \in \mathcal{S}_{g}^{(\alpha)}(p, k, \lambda, \mu, b, \beta, m, n)$. This completes the proof of Theorem 15. 
Remark 16. If we set $\lambda=\mu=0$ and $\beta=1$ in Theorem 15 , then we have [2, Theorem 5].

The proof of Theorem 17 (based upon Definition 14) is similar to that of Theorem 15. Therefore we omit the details involved.

Theorem 17. If $h \in \mathscr{P}_{g}(p, k, \lambda, \mu, b, \beta, m, n ; \gamma)$ and

$$
\begin{aligned}
\alpha= & p-\frac{\delta}{k^{q+1}} \\
& \times\left((k-n)_{m}\left[\gamma(k)_{n}-(\gamma-1)(p)_{n}\right] \psi(k) b_{k}\right) \\
\times & \left((k-n)_{m}\left[\gamma(k)_{n}-(\gamma-1)(p)_{n}\right] \psi(k) b_{k}\right. \\
& \left.\quad-(p-m)_{n}\left[|b|-1+(p)_{m} \psi(p)\right]\right)^{-1}
\end{aligned}
$$

then

$$
\mathcal{N}_{k, \delta}^{q}(h) \subset \mathscr{P}_{g}^{(\alpha)}(p, k, \lambda, \mu, b, \beta, m, n ; \gamma),
$$

where $\psi$ is defined by (15).

Remark 18. If we set $\lambda=\mu=0$ and $\beta=1$ in Theorem 17, then we have $[2$, Theorem 6].

\section{Acknowledgment}

The present investigation was supported by the Kocaeli University under Grant HD 2011/22.

\section{References}

[1] A. O. Mostafa and M. K. Aouf, "Neighborhoods of certain $p$ valent analytic functions with complex order," Computers \& Mathematics with Applications, vol. 58, no. 6, pp. 1183-1189, 2009.

[2] H. M. Srivastava, S. S. Eker, and B. Şeker, "Inclusion and neighborhood properties for certain classes of multivalently analytic functions of complex order associated with the convolution structure," Applied Mathematics and Computation, vol. 212, no. 1, pp. 66-71, 2009.

[3] J. K. Prajapat, R. K. Raina, and H. M. Srivastava, "Inclusion and neighborhood properties for certain classes of multivalently analytic functions associated with the convolution structure," Journal of Inequalities in Pure and Applied Mathematics, vol. 8, no. 1 , article 7, 8 pages, 2007.

[4] H. M. Srivastava and S. Bulut, "Neighborhood properties of certain classes of multivalently analytic functions associated with the convolution structure," Applied Mathematics and Computation, vol. 218, no. 11, pp. 6511-6518, 2012.

[5] R. M. Ali, M. H. Khan, V. Ravichandran, and K. G. Subramanian, "A class of multivalent functions with negative coefficients defined by convolution," Bulletin of the Korean Mathematical Society, vol. 43, no. 1, pp. 179-188, 2006.

[6] B. A. Frasin and M. Darus, "Integral means and neighborhoods for analytic univalent functions with negative coefficients," Soochow Journal of Mathematics, vol. 30, no. 2, pp. 217-223, 2004. 


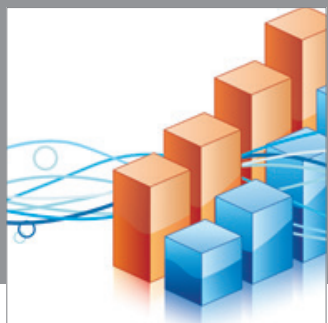

Advances in

Operations Research

mansans

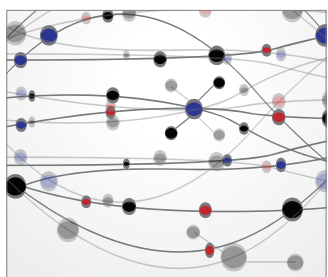

The Scientific World Journal
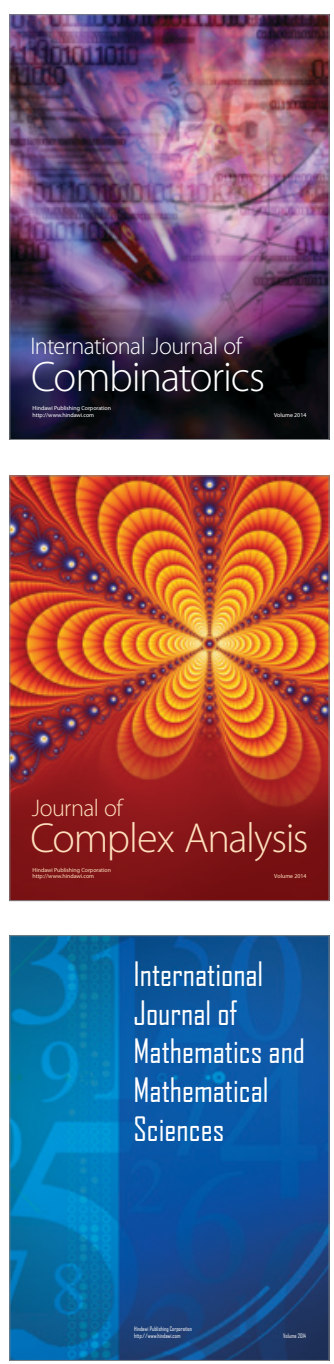
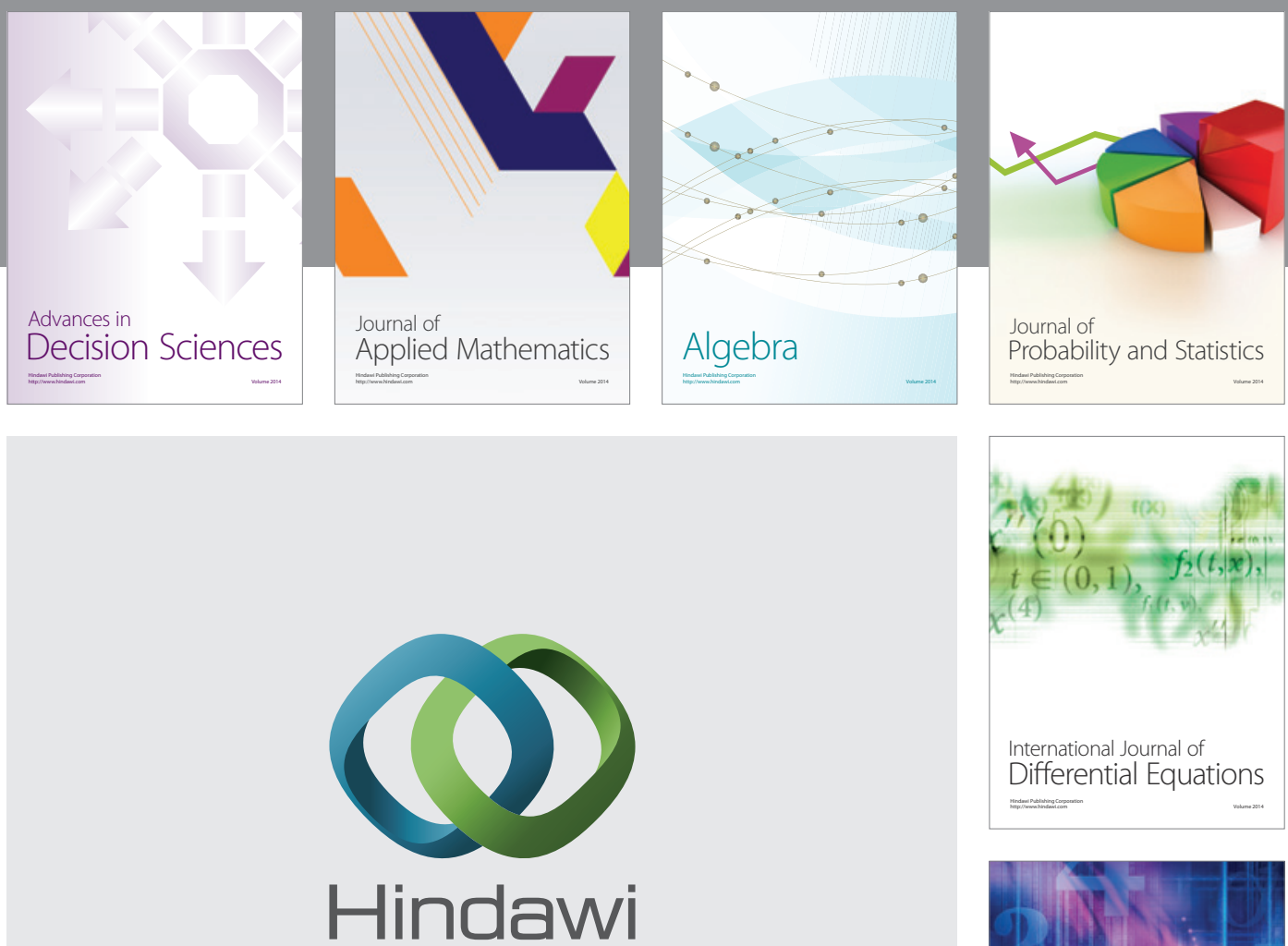

Submit your manuscripts at http://www.hindawi.com
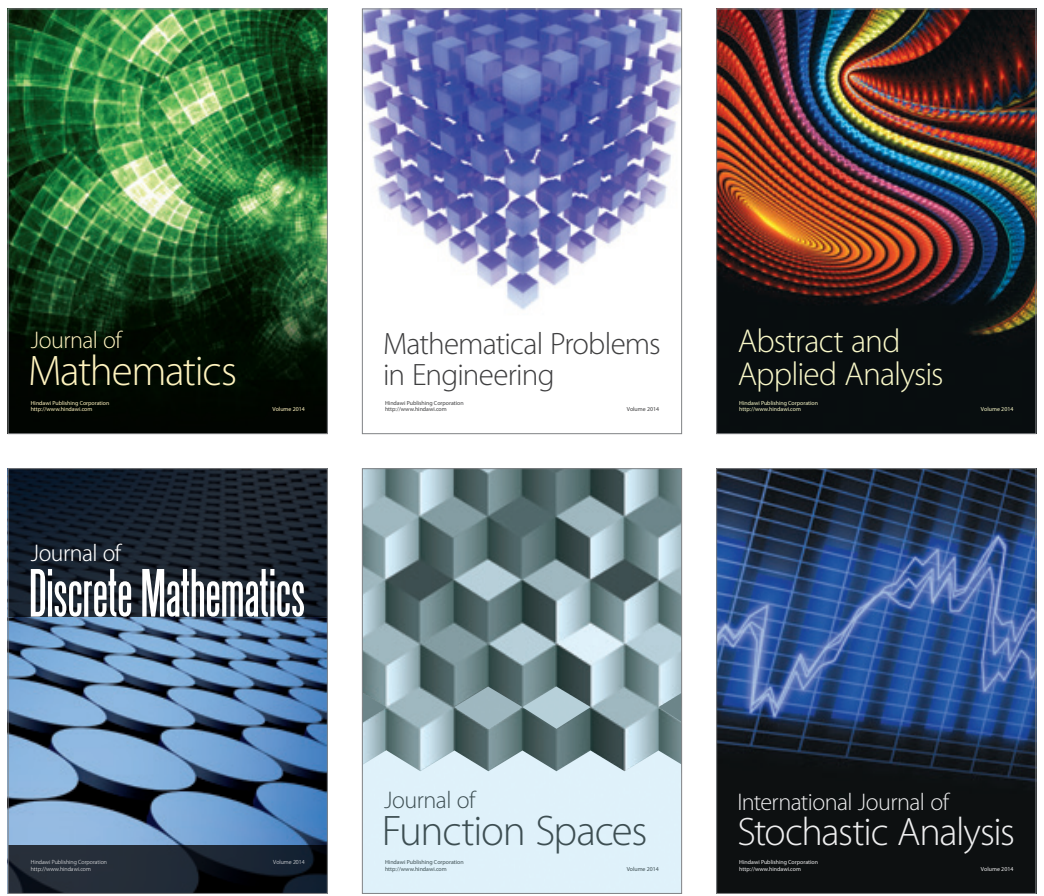

Journal of

Function Spaces

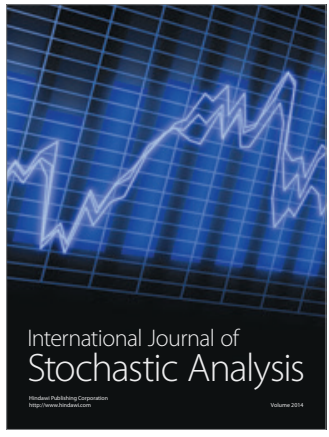

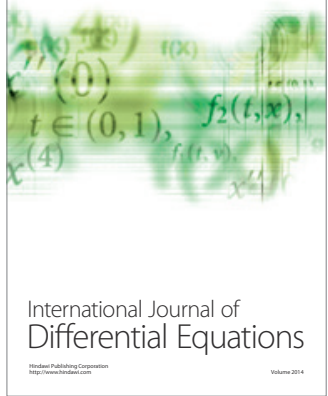
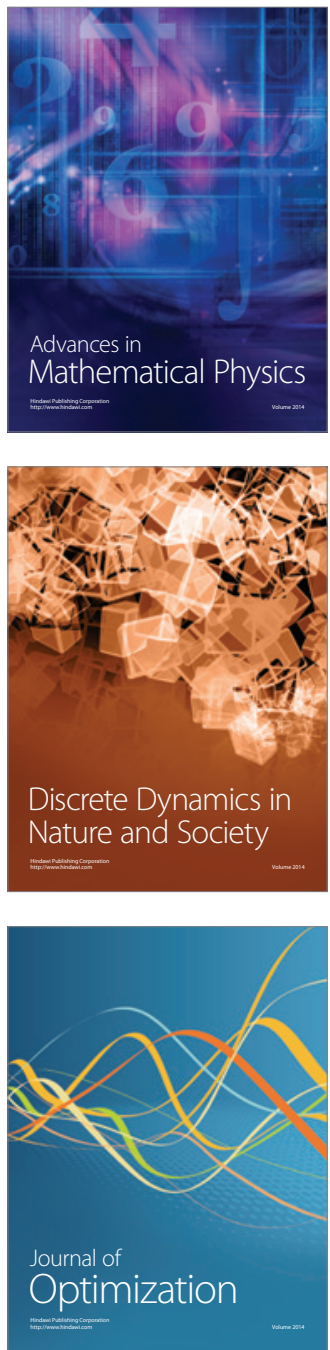